\title{
Memory Accuracy for Emotional and Neutral Narratives: Investigating the Influence of Suggestive Misinformation on the Frequency of False Memories
}

\author{
Laura E. Paige and Elizabeth A. Kensinger \\ Psychology Department \\ Boston College \\ Chestnut Hill, Massachusetts 02467 USA
}

Received: September 19, 2012

Accepted: October 25, 2012

\begin{abstract}
Memory is a reconstructive process, impressionable and not always vigilant in detecting devices working against its accuracy. False memory occurs when memory for an event is infiltrated by new information or alterations are made to the information that was previously stored. The present study investigated the effect of emotional valence on false memory occurrence through the misinformation effect - memory errors that arise as a consequence of exposure to misleading information - by presenting participants with narratives in one of three valence conditions (negative, positive, neutral) and subjecting them to suggestive questions providing credulous misinformation following a one-hour delay. Results revealed that when collapsing across negative and positive conditions, overall false memory occurrences were significantly reduced for these narratives compared to the neutral condition. Some of the effects of emotion on memory accuracy were more pronounced for negative information than for positive or neutral information; response rates in the negative condition, as compared to the two other valence conditions, revealed an increase in hits and a decrease in false alarms suggesting negative valence enhances discriminability.
\end{abstract}

\section{INTRODUCTION}

While much of memory research focuses on the vast capacities and triumphs of our brain to encode and recall, the past decades have seen a growing interest in how the mind fails- its pitfalls and limitations. Memory is malleable and situations, stories, details, and events that seem genuine can infiltrate memory with tools as simple as suggestions. False memories can occur in either remembering events that never happened or remembering them significantly different from the way they actually occurred [1]. There are various means by which false memories are elicited, but much research has focused on the role of misinformation disguised through suggestion.

The misinformation effect is the impairment in memory for the past as a result of exposure to misleading information
[2]. Once a novel phenomenon to the field, substantial research on the effect has provided a significant history in considering the conditions, possible resistance, susceptibility, and degree to which memory is malleable in altering original traces [2]. The nature of misinformed unreal memories is incredibly difficult to pinpoint- while verbal descriptions of unreal memories have shown to be longer, with more verbal ambiguities and fewer sensory details, there is enough variability to prevent any single marker to distinguish a true from false memory [2].

To understand why misinformed suggestions could impact memory, it is important to recognize that rarely does a memory contain all of the details of a prior event. Suggestibility in memory is our tendency to incorporate misleading information from external sources into personal recollections, typically through 
misattributions [3]. The idea of the "gist" of the stimuli is used by both the common man and cognitive psychologist alike in considering the degree of memory specificity. Brainerd and Reyna [4] presented a new perspective on this framework of cognition. Their fuzzy trace theory is an understanding of how intuition is taken as the main operation for cognition, rather than deductive logic. It has been shown that our cognition has a tendency to rely on heuristics, or simple techniques, shortcuts, and strategies known to assist in problem solving. One of the fundamental elements of the theory is that in encoding, we reduce information to its essencesenses, patterns, and gists. Therefore, in doing so, there is an inclination to reason and remember more of the fuzzy elements than verbatim traces. The theory ultimately boils down to being as near as possible in tasks and how this correlates in supporting accurate performance. In relation to false memory formation, the fuzzy trace theory emphasizes that information processing is not always precise. Details may not be encoded, and this leaves us open to accepting others' suggestions, correct or incorrect, for what might have occurred.

A crucial feature in false memory occurrence is distinguishing the conditions in which the effect is most robust- or the means by which a person fails to detect the discrepancy [2]. Prior findings have supposed that repeated exposure to misinformation can strengthen the occurrence of false memories as it generates a false heightened sense of confidence in participants remembering suggested events from videos [5]. Reflective and repetitive processes in suggestive misinformation will create an increasingly elaborate, detailed, and realistic image [5]. In investigating individual differences in false memory frequency through suggestibility and misinformation, previous work has shown that participants who score lower on measurements of extraversion are more susceptible to creating false childhood memories [6]. Moreover, false memory frequency has been positively correlated with the openness dimension, modesty, and altruism from the NEO Personality Inventory [7].

Additional research has shown that the presence of a confederate, a disguised research assistant trained to provide misleading information and suggestions during the task, reveals the effects of conformity on recognition judgments [8]. Socially encountered post-event misinformation has typically shown larger effects on participants' recognition reports for new rather than old items, suggesting memory is more susceptible to accepting information about items for which no actual memory exists [9].

The effects of emotion on memory have been shown to enhance subsequent recognition of specific visual details [10]. Consequently, valence can produce lingering effects on memory in which heightened attention toward emotional material diminishes consolidation of contextual details, but the debate regarding valence and trade-offs between content and context continues. Cook, Hicks, and Marsh [11] revealed results of a source-monitoring deficit for valenced words in comparison to neutral words. While these results were novel in comparison to previous studies consistently exhibiting enhanced source memory on valenced items, the study took into account the more rich and complex emotional characteristics of everyday life. As such, it suggests that while emotional events are actually being experienced, a narrowing of the focus of attention occurs resulting in a memory trade-off between central details and peripheral ones.

To date, research concerning the effects of emotion on memory and suggestive misinformation has focused upon recall image memory placing participants in one of three conditions- items of negative, positive, or neutral content- with a significant delay between initial encoding of the valenced video or scene and the subsequent presentation of misleading information later. In real-life application, recall from eyewitness testimony to clinical convention would not be devoid of emotional content. While much research has demonstrated the misinformation effect, this present study is novel in the fact that it uses narratives and is one of the few to consider the supplementary influence of emotional content on suggestibility to misinformation. In considering the effect on memory for emotional scenes, Porter et al [12] took into account the impact on general memory as well as susceptibility. Their findings reveal 
that negative scenes were not only less accurately recalled, but also negative emotion heightened suggestibility through misinformation. However, there is still much ground for debate when considering confounding factors such as retrieval, delay and deterioration over time, and intensity.

Compliant with the framework of the fuzzy trace theory, by merely grasping the "gist" of the information, there is an inclination to seek a connection across distinct features that share meaning, or in this case, valence. In condensing information, recent studies have shown that false memory frequency increases, and net accuracy decreases, with both age and negatively valenced materials [13]. As a more salient entity, items of negative valence are more likely to be the focus of processing- creating stronger gist memories and consequently higher false alarm rates for negative, rather than positive, distractors [13].

The present study examined the influence of suggestive misinformation on the frequency of false memories with the use of narratives. Preceding research involving the frequency of false memories through suggestibility had used images or videos as the initial event and participants received misinformation either via confederate or suggestive questionnaire. However, we were interested in the effect of suggestive misinformation on the frequency of false memories using narratives as the vehicle and a delay of one hour before being subjected to suggestive questions regarding the narratives. Our primary hypothesis was that suggestive misinformation facilitates the frequency of false memories.

\section{METHOD}

\section{a. Participants}

Participants for this study included 11 male and 34 female right-handed, native English speakers. All participants had normal or corrected-to-normal vision, were in good health, and had no prior experience with the study or stimuli. The sampling pool was the student body population of Boston College ranging in age from $18-25$ years old $(M=19.58, S D=1.95)$. Recruitment was carried out online through the use of the BC Sona System. Participants received either cash (\$15) or course credit (1.5) for their compensation. All testing was completed in accordance with the Boston College Institutional Review Board.

\section{b. Stimuli and Procedure}

A series of emotional narratives were used as originally presented in Bishop, Dalgleish, and Yule [14]. While the original narratives were comprised of text and pictures, only the text was used in the present study since the current focus was the creation of false memories from text. The nine narratives consisted of three core stories ("park", "beach", "going home") that each included three separate second paragraphs differing in valence (negative, positive, neutral). Therefore, while the core story remained the same across versions, changing various key words or phrases allowed for differing emotional tones while maintaining the same overall structure. In the Bishop, Dalgleish, and Yule [14] study, each of the nine narratives was rated for how pleasant/unpleasant they were to confirm the success of the valence manipulation. Each of the narratives was between 390 and 460 words and versions did not differ in length by more than 5 words. In the present study, the nine narratives were kept the same with the exception of a few words that were converted from British to American English (i.e. "conkers" to "acorns", "mum" to "mom"). A shorter, slightly positive, practice story was given first as an example of the types of narratives to follow.

Participants were tested individually. Each participant was assigned to a single valence category (negative, positive, or neutral) and was presented with a hard copy of each of the stories in that valence condition. Narrative order was alternated across participants. Participants read each narrative printed on a piece of paper, and materials were collected immediately upon their completion.

During a one hour delay, participants were administered the Positive and Negative Affect Scale (PANAS; [15]) among other cognitive measures. The PANAS is a self-report assessment that measures the present degree of positive and negative mood. 


\begin{tabular}{|cccc|}
\hline Cognitive Measure & Negative $(\mathbf{N}=\mathbf{1 5})$ & Positive $(\mathbf{N}=\mathbf{1 5})$ & Neutral $(\mathbf{N}=\mathbf{1 5})$ \\
\hline BADS-DEX & 16.0 & 15.47 & 17.87 \\
\hline PANAS (PA) & 35.67 & 36.27 & 36.47 \\
\hline PANAS (NA) & 14.8 & 15.6 & 17.13 \\
\hline BDI & 3.53 & 3.13 & 5.2 \\
\hline BAI & 6.0 & 7.93 & 8.0 \\
\hline Shipley & 32.53 & 31.93 & 32.33 \\
\hline
\end{tabular}

Table 1. Average Participant Scores on Cognitive Measures by Condition. Note: The PANAS calculation is comprised of scores for both positive affect (PA) and negative affect (NA).

After the delay, a memory questionnaire was administered using the program E-Prime (Psychology Software Tools, Pittsburgh, PA). The questionnaire, constructed specifically for this experiment, consisted of 135 questions, with 45 questions about each story. All questions for a particular narrative were presented together. Analogous to the organization of the narratives with three "core" stories that differed only in the valenced content of their second paragraph, questionnaires for a particular "core" story contained 33 of the same questions in sequential order for narrative $A, 35$ for $B$, and 34 for $C$. The remaining questions differed depending on the valence condition. Questions appeared on the screen in a pre-set sequential order for an infinite amount of time until a response was made. All questions required a "yes" or "no" response recorded through key press. A short trial questionnaire regarding the practice story each participant read was first given to exemplify the format of the questionnaire.

\section{c. Questionnaire Scoring}

Questionnaires were scored for false memories in two ways. "Omnibus false memories" were considered to be any question for which the correct answer was "no" but the response was "yes." "Misinformation false memories" were a subset of these instances. The questionnaire was designed to include 10 questions in which the correct answer was "no" but prior suggestive questions increased the likelihood of a participant answering "yes." If participants responded "yes" to one of these questions, this was scored not only as an omnibus false memory but also as a "misinformation false memory." Suggestive questions were based off of similar ones seen on Wechsler's Logical Memory cognitive task (WMS-IV; [16]), defined as one that implies a certain answer or presents a presupposition as an accepted fact.

Example of initial suggestions and "misinformation false memory" question (correct answer in parentheses):

1. There were not a lot of children on the swings. (YES)

2. Children were waiting to use the slide. (YES)

3. Jane had just come off the slide.

(NO; misinformation false memory)

Above, in reference to the "park" story, the first two questions are explicitly stated in the text- few children were on the swings and many were waiting to use the slide. The story also reads, "Jane had just come off the swings." By correctly stating that few children were on the swings and that children were waiting to use the slide, the questionnaire was designed to suggest to participants that the misinformation presented in question 3 was plausible and perhaps true. The "misinformation false memory" questions, like this one, were always preceded by at least one suggestive (correct) statement. To ensure a sufficient number of false endorsements for analysis, each questionnaire portion consisted of 10 "misinformation false memory" questions for a total of 30 attempted misinformationinduced false memories per participant. In addition to examining the false memories (i.e., incorrect "yes" responses), questionnaires were also scored for the number of "hits" (correct "yes" responses), misses (incorrect "no" responses), and correct rejections (correct "no" responses). 


\begin{tabular}{|lcc|c|}
\hline Condition & N & Hits & False Alarms \\
\hline Negative & 15 & $0.83(0.082)$ & $0.28(0.10)$ \\
\hline Positive & 15 & $0.77(0.10)$ & $0.28(0.081)$ \\
\hline Neutral & 15 & $0.83(0.067)$ & $0.35(0.082)$ \\
\hline
\end{tabular}

Table 2. Average statements correctly and incorrectly endorsed from each valence condition.

These values were also used to compute discrimination (d') scores, or participants' ability to distinguish correct from incorrect statements.

\section{RESULTS}

\section{a. Supplemental Results}

During the 60-minute delay between reading the narratives and answering the subsequent questionnaire on the computer, participants completed a packet containing cognitive tasks of various measures. Individual scores were calculated for: BADSDEX [17], PANAS [15], BDI [18], BAI [19], Shipley Vocabulary Test [20] (see Table 1 for means). Between-group analysis of cognitive scores was not significant: $F(2,42)$ $<1.363, p>0.267$

\section{b. Recognition Results: Total True and False Endorsements.}

For each participant, the total number of hits, correct rejections, misses, and false alarms were determined and recognition rates calculated for each valence group. A one-way ANOVA examined the effect of valence (negative, positive, neutral) and endorsement type (true = hits, false = false alarms) on these frequencies. Results revealed there was a significant main effect of endorsement, $F(1,42)=722.201, p<$ 0.05 . Participants across all valence groups had a greater proportion of hits than false alarms (see Table 2 for means). There was no significant interaction between endorsement and valence condition, $F(2,42)$ $=1.481, p>0.239$.
Although there was no significant interaction in this ANOVA, a planned contrast comparing the false alarms for all participants in an emotion condition (collapsing the negative and positive narratives) to those in the neutral condition revealed a significant effect, $t(43)=2.466, p$ $<0.05$. Participants in the neutral valence condition had a higher false alarm rate $(\mathrm{M}=0.35, \mathrm{SE}=0.021)$ than participants in an emotion condition ( $M=0.28, S E=0.017)$. An independent t-test confirmed there was no significant effect between the negative and positive conditions, $\mathrm{t}(28)=0.115, \mathrm{p}>0.909$. However, one possible caveat to consider is the effect collapsing across valence has on power-there are now 30 participants in the emotion condition and 15 in the neutral condition.

c. Recognition Results: False

Endorsements related to Misinformation Effect.

In the prior section, false alarms included all instances in which the correct answer was "no" but the participant responded "yes." Next, we examined the effect of valence on false endorsements related to the misinformation effect, seen as incorrect endorsements to the 30 "misinformation false memory" questions that were preceded by leading questions.

A one-way ANOVA examined the effect of valence (negative, positive, neutral) on false alarm rate related to misinformation. This analysis did not reveal a significant effect of valence, $F(2,42)=2.487, p>0.095$. However, a planned contrast comparing these false memories for the participants in an emotion condition (collapsing negative 


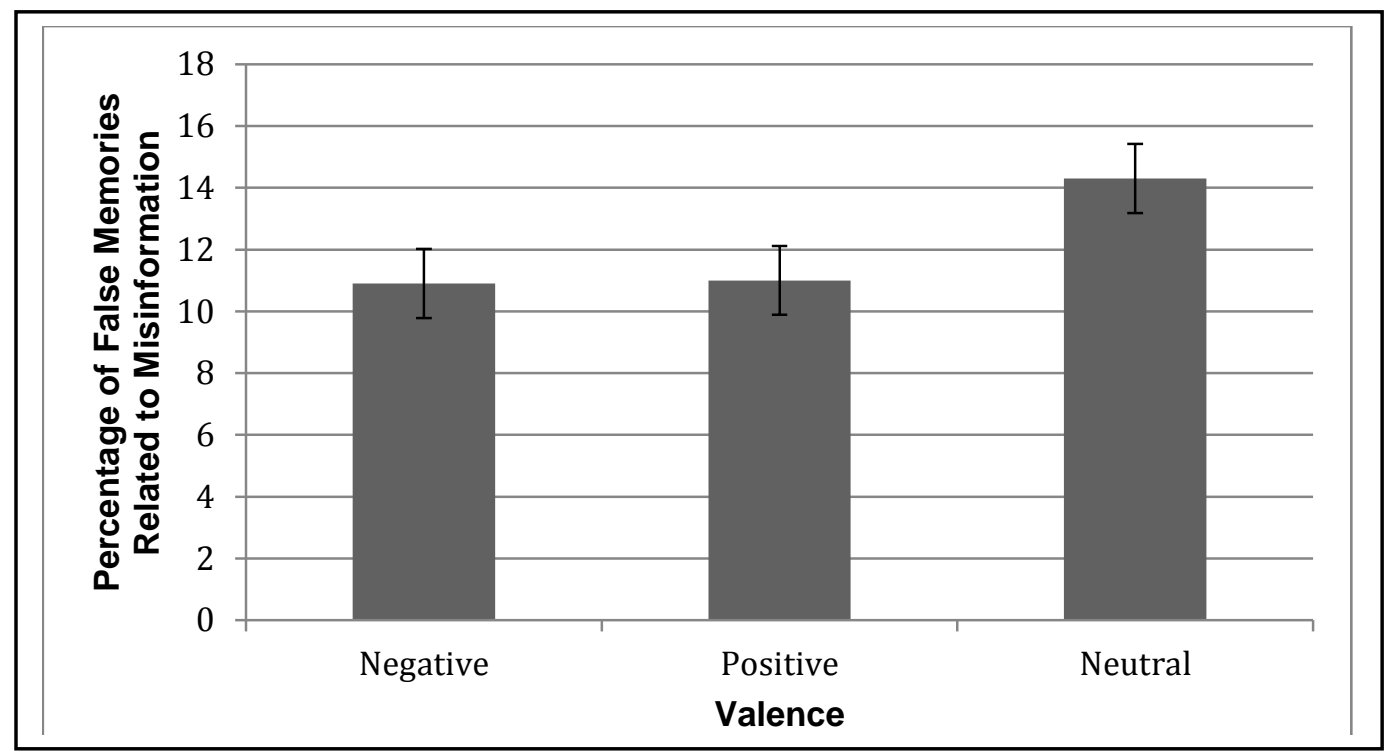

Figure 1. Comparison of misinformation rate within each valence condition. This chart illustrates that, of the false memories people have, a greater percentage stem from misinformation in the neutral condition than in the negative or positive condition.

and positive) to those in the neutral condition revealed a significant difference, $\mathrm{t}(43)=2.255, \mathrm{p}<0.05$. Participants who read a neutral narrative had a higher false alarm rate related to misinformation $(\mathrm{M}=0.14, \mathrm{SE}=0.010)$ than did participants who read an emotional narrative $(\mathrm{M}=0.11$, $\mathrm{SE}=0.0091$ ).

Although the above analyses focused on the raw number of false alarms related to misinformation, another way to examine the robustness of the misinformation effect is to consider the proportion of omnibus false alarms that are related to misinformation (Figure 1). For each participant, a proportion was calculated by dividing the total possible number of misinformation false alarms by the total number of omnibus false alarms. A oneway ANOVA examined the effect of valence (negative, positive, neutral) on this proportion of false alarms related to misinformation. As with the raw false alarm scores, this analysis did not reveal a significant effect of valence, $F(2,42)=2.263$, $\mathrm{p}>0.117$. However, a planned contrast comparing this proportion for participants in an emotion condition (collapsing negative and positive) to participants in the neutral condition revealed a significant difference, $\mathrm{t}(43)=2.148, \mathrm{p}<0.05$. A larger proportion of the false alarms were caused by misinformation in the neutral condition $(\mathrm{M}=0.21, \mathrm{SE}=0.013)$ than in the emotion condition $(\mathrm{M}=0.17, \mathrm{SE}=0.0099)$.

d. Recognition Results: d'

d' scores for each of the three valence conditions were calculated on a subject-by-subject basis (Figure 2). $d$ ' is a measurement from signal detection theory that considers hits as a response to a "signal" and false alarms/false memories as a response to "noise;" d' represents the ability for participants to discriminate the "signals" (hits) from the "noise" (false alarms) [21]. Discrimination is better for participants in the negative condition than the neutral or positive condition. A one-way ANOVA examined the effects of valence (negative, positive, neutral) on discrimination. This analysis did not reveal a significant effect of valence, $F(2,42)=$ 1.728, $p>0.190$.

\section{DISCUSSION}

a. Summary of Results

In the present study, our interest was determining whether suggestive misinformation facilitates the formation of 


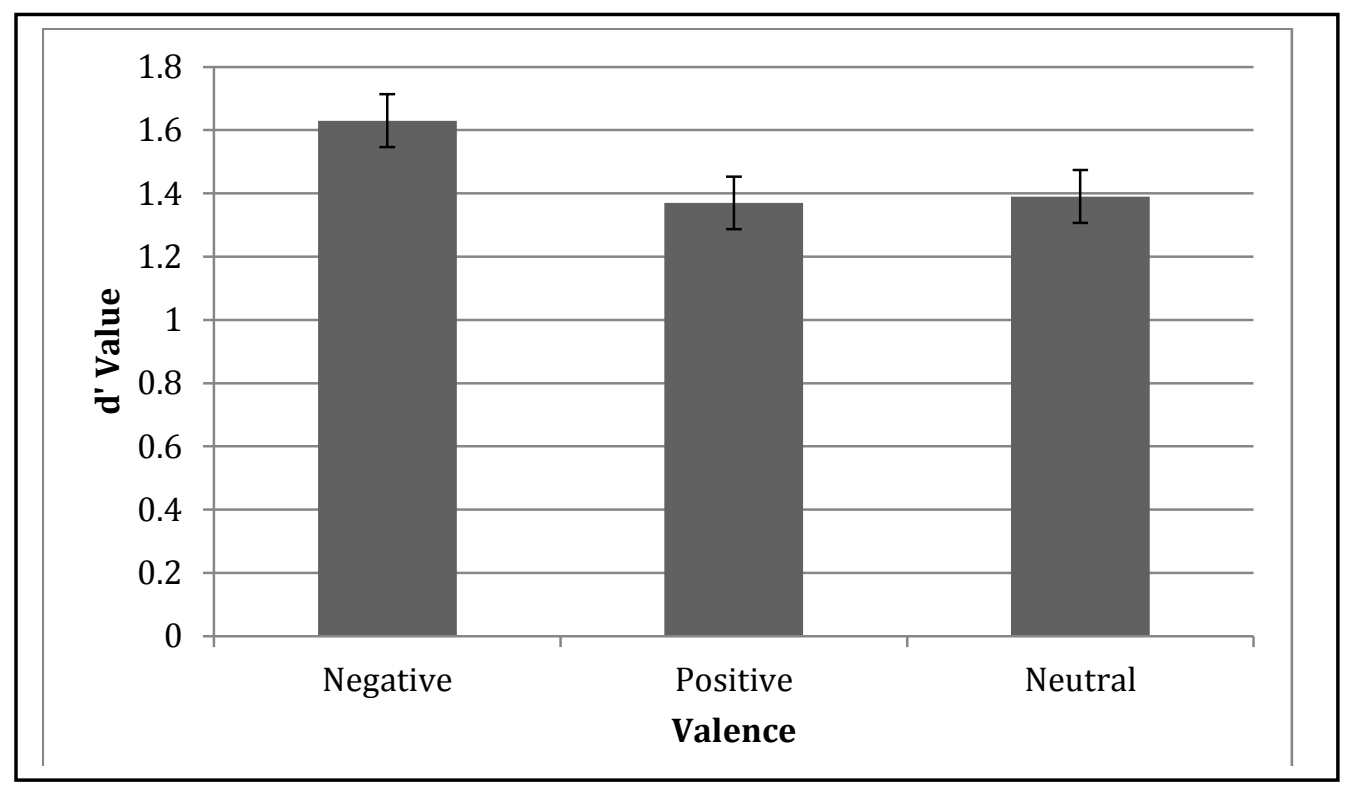

Figure 2. d' values for the three valence conditions- negative (1.63), positive (1.37), neutral (1.39). This reveals that while people are more likely to incorporate false memories for items of neutral valence, negative valence actually enhances discriminability. The large d'value for the negative condition exemplifies that people were better at discriminating hits from false alarms/false memories.

false memories. The key finding was that when collapsing across emotion, people are more likely to incorporate false memories for neutral stimuli. Additionally, we were interested in the participants' overall discriminative criteria in responding to each question. The d' analyses reveal improved discrimination for emotional content than neutral content, with discrimination being best for negative narratives.

When looking specifically at the "misinformation false memory" questions, there was a significant difference between the false alarm rates for emotion (negative and positive) versus neutral, with more misinformed false memories occurring when stimuli are of neutral valence rather than positive or negative, and with a larger proportion of the omnibus false memories in the neutral condition coming from the presence of misinformation. We can therefore suggest that the omnibus false alarm results are driven by misinformed false alarms.

\section{b. Relation to prior research}

False memory formation is the failure to attribute information to the correct source or there is insufficient information available for discrimination in either remembering events that never happened or remembering them differently from the way they actually happened [1,3]. Previous research has shown effects of picture superiority in free recall versus semantic [22], but even with the substantial accuracy of image memory, it is still highly susceptible to false recognition, high confidence, and a strong sense of familiarity for objects never shown in the images [23]. Contradictory research has also shown that when it comes to details and complexity, image memory declines [24], suggesting the possibility that a reciprocal relationship between images and text could be seen in false memory occurrence if frequency is dependent on stimulus type. The present study is novel in that it investigated this possibility by focusing on the frequency of false memory occurrence with narratives through the misinformation effect- impairment in memory for the past through exposure, in this case suggestive questions, to misleading information [2]. The designated "misinformation false memory" questions are preceded by suggestive questions all of which are coherent and plausible. There are 
many vehicles by which false memories can be elicited ranging from failures in source monitoring to merely settling for the "gist." Through the work of Brainerd and Reyna [4], the fuzzy trace theory demonstrates our own reliance on simple techniques, shortcuts, strategies, or even "gut-feelings" in order to be as near as possible.

While previous research has revealed that negative emotion enhances subsequent recognition of specific visual details [10], other research has suggested that negative emotion can impair memory for source details (e.g., Otani et al. [25]). It was therefore an open question as to how misinformed false memory frequency would be affected by the valence of narratives. Our results signify that emotion does enhance recognition ability in verbal memory- people are more susceptible to misinformed false memories with neutral stimuli. In relation to discriminability, this research supports the notion that emotion enhances recognition and increases our own vigilance, especially if the information is negative. The fact that negative emotion may enhance discriminability more than positive emotion is consistent with prior evidence that negative emotion may be more likely than positive emotion to encourage an item-specific or analytic form of processing, thereby reducing false memories [26].

Although our results suggest that without a specific emotional connotation, participants are merely seeking for the "gist" of the more mundane narratives, some previous research has proposed the contrary in which negative valence may lead to a more liberal response bias. Results revealed falsification was greatest for negative, followed by neutral and then positive materials, suggesting that people are less able to use precise memories of studied negative items to suppress memory errors [27]. However, the disparity in methodology in comparison to our study must be noted. It has been suggested that negative valence enhances the familiarity of the semantic content of critical distractors while simultaneously reducing the ability to suppress errors with verbatim traces [27]. Although that opposition may be critical in studies of unrelated word lists, where semantic familiarity may lead to associative false memories, in the present study, the tendency for negative valence to enhance familiarity of the semantic content may actually be consistent with our results that negative valence revealed an increased number of hits and a decreased number of false alarms. That is, in a narrative context, familiarity of semantic content may improve, rather than diminish, discriminability.

\section{c. Implications}

Ultimately, false memories may be complete, emotional, or self-participatory experiences resulting from social demands to remember, memory construction by imagination, or even encouragement to be ignorant of whether the constructions are real or not [28]. Research in the field, while altogether harmless to participants, transcends into much greater and more disturbing real world application ranging from false traumatic memories in adults [29] to the more serious consequences seen in eyewitness influence and false confessions in the courtroom. Witnesses who are highly confident are no more accurate than witnesses who are less confident- and confidence can only be inflated with the addition of a second witness or rehearsal prior to taking the stand. While witnesses could fall prey to the assurance of an administrating officer, the court verdict may or may not impact their lives. What is more disturbing is the impact that suggestion in the courtroom plays in the facilitation of false memories leading to false confessions. They may occur to alleviate emotional stress, social pressures, or abuse, but our ability to mistakenly believe in our own guilt can occur without significant coercion and simply with the assistance of suggestion [3, 30]. While investigative research into the formation of false memories continues, the major questions that lie ahead include what kinds of false memories are likely to form and who is most vulnerable?

In the greater realm of psychological evidence, our results do not directly inform current debates and controversies regarding false memories induced in therapy, trauma, or the courtroom. We must not be naive in assuming that people merely claiming to vividly remember details of an event, image, or text is conclusive enough as evidence. However, while our results are not directly transferable, the present findings exemplify 
the way in which innocuous false memories are formed with tools as simple as suggestion- even neutral in content - in our everyday lives. Ultimately, the present study further solidifies the existence of false memory formation in semantic memory, specifically through the vehicle of suggestive misinformation. While our results were collected in a controlled laboratory setting, using harmless narratives of differing emotional valence, and a subject pool of college students, these results are fundamental elements of the greater domain of false memory occurrence.

\section{REFERENCES}

1. Roediger III, H.L. \& McDermott, K.B. (1995). Creating false memories: remembering words not presented in lists. Journal of Experimental Psychology, 21, 803-814.

2. Loftus, E. (2005). Planting misinformation in the human mind: a 30 year investigation of the malleability of memory. Learning \& Memory, 12, 361366.

3. Schacter, D.L. (2001). The seven sins of memory: how the mind forgets and remembers. Boston: Houghton Mifflin.

4. Brainerd, C.J. \& Reyna, V.F. (1990). Gist is the grist: fuzzy-trace theory and the new intuitionism. Developmental Review, 10, 3-47.

5. Zaragoza, M.S. \& Mitchell, K.J. (1996). Repeated exposure to suggestion and the creation of false memories. Psychological Science, 7, 294-300.

6. Porter, S., Birt, A.R., Yuille, J.C., \& Lehman, D. (2000). Negotiating of false memories: interviewer and remember characteristics relate to memory distortion. Psychological Science, 11, 513-516.

7. Liebman, J.I. et al. (2002). Cognitive and psychosocial correlates of adults' eyewitness accuracy and suggestibility. Personality and Individual Differences, 33, 49-66.

8. Brown, C. \& Schaefer, A. (2010). The effects of conformity on recognition judgments for emotional stimuli. Acta Psychologia, 133, 38-44.

9. Wright, D.B., Mathews, S.A., \& Skagerberg, E.M. (2005). Social recognition memory: the effect of other people's responses for previously seen and unseen items. Journal of Experimental Psychology: Applied, 11, 200-209.

10. Kensinger, E.A., Garoff-Eaton, R.J., \& Schacter, D.L. (2006). Memory for specific visual details can be enhanced by negative arousing content. Journal of Memory and Language, 54, 99-112.

11. Cook, G.I., Hicks, J.L., \& Marsh, R.L. (2007). Source monitoring is not always enhanced for valenced material. Memory \& Cognition, 35, 222-230.

12. Porter, S., Bellhouse, S., McDougall, A., ten Brinke, L., \& Wilson, K. (2010). A prospective investigation of the vulnerability of memory for positive and negative emotional scenes to the misinformation effect. Canadian Journal of Behavioural Science, 42, 55-61.

13. Brainerd, C.J., Holliday, R.E., Reyna, V.F., Yang, Y., \& Toglia, M.P. (2010). Developmental reversals in false memory: effects of emotional valence and arousal. Journal of Experimental Child Psychology, 107, 137-154.

14. Bishop, S.J., Dalgleish, T., \& Yule, W. (2004). Memory for emotional stories in high and low depressed children. Memory, 12, 214-230.

15. Watson, D., Clark, L.A., \& Tellegen, A. (1988). Development and validation of brief measures of positive and negative affect: the PANAS scales. Journal of Personality and Social Psychology, 47, 1063-1070.

16. Wechsler, D., Holdnack, J.A., \& Drozdick, L. W. (2009). Wechsler memory scale, fourth edition, technical and interpretive manual. San Antonio, TX: NCS Pearson, Inc.

17. Wilson, B.A., Alderman, N., Burgess, P.W., Emslie, H.C., \& Evans, J.J. (1996). The behavioural assessment of the dysexecutive syndrome. Flempton, Bury St. Edmunds, England: Thames Valley Test Company.

18. Beck, A.T., Ward, C.H., Mendelson, M., Mock, J., Erbaugh, J. (1961). An inventory for measuring depression. Arch. Gen. Psychiatry, 4, 561-71.

19. Beck, A.T., Epstein, N., Brown, G., \& Steer, R. A. (1988). An inventory for measuring clinical anxiety: psychometric 
properties. Journal of Consulting and Clinical Psychology, 56, 893-897.

20. Shipley, W.C. (1986). Shipley Institute of Living Scale, Los Angeles: Western Psychological Services.

21. Wilson, T. \& Swets, J.A. (1954). A decision-making theory on visual detection. Psychological Review, 61, 401-409.

22. Paivio, A. \& Csapo, K. (1973). Picture superiority in free recall : imagery or dual coding? Cognitive Psychology, 5, 176-206.

23. Weinstein, Y. \& Shanks, D.R. (2010). Rapid induction of false memories for pictures. Memory, 18, 533-42.

24. Goldstein, A.G. \& Chance, J.E. (1970). Visual recognition memory for complex configurations. Perception and Psychophysics, 9, 237-241.

25. Otani, H., Libkuman, T.M., Goernert, P. N., Kato, K., Migita, M., Freehafer, S.E., \& Landow, M.P. (2012). Emotion, directed forgetting, and source memory.
British Journal of Psychology, 103, 343358.

26. Storbeck, J. \& Clore, G.L. (2005). With sadness comes accuracy; with happiness, false memory: mood and the false memory effect. Psychological Science, 10, 785-791.

27. Brainerd, C.J., Stein, L.M., Silveira, R.A., Rohenkohl, G., \& Reyna, V.F. (2008). How does negative emotion cause false memories? Psychological Science, 19, 919-925.

28. Loftus, E. (1997). Creating false memories. Scientific American, 277, 7075.

29. Loftus, E.F. \& Pickrell, J. (1995). The formation of false memories. Psychiatric Annals, 25, 720-725.

30. Kassin, S.M. \& Kiechel, K.L. (1996). The social psychology of false confessions: compliance, internalization, and confabulation. Psychological Science, 7, 125-128.

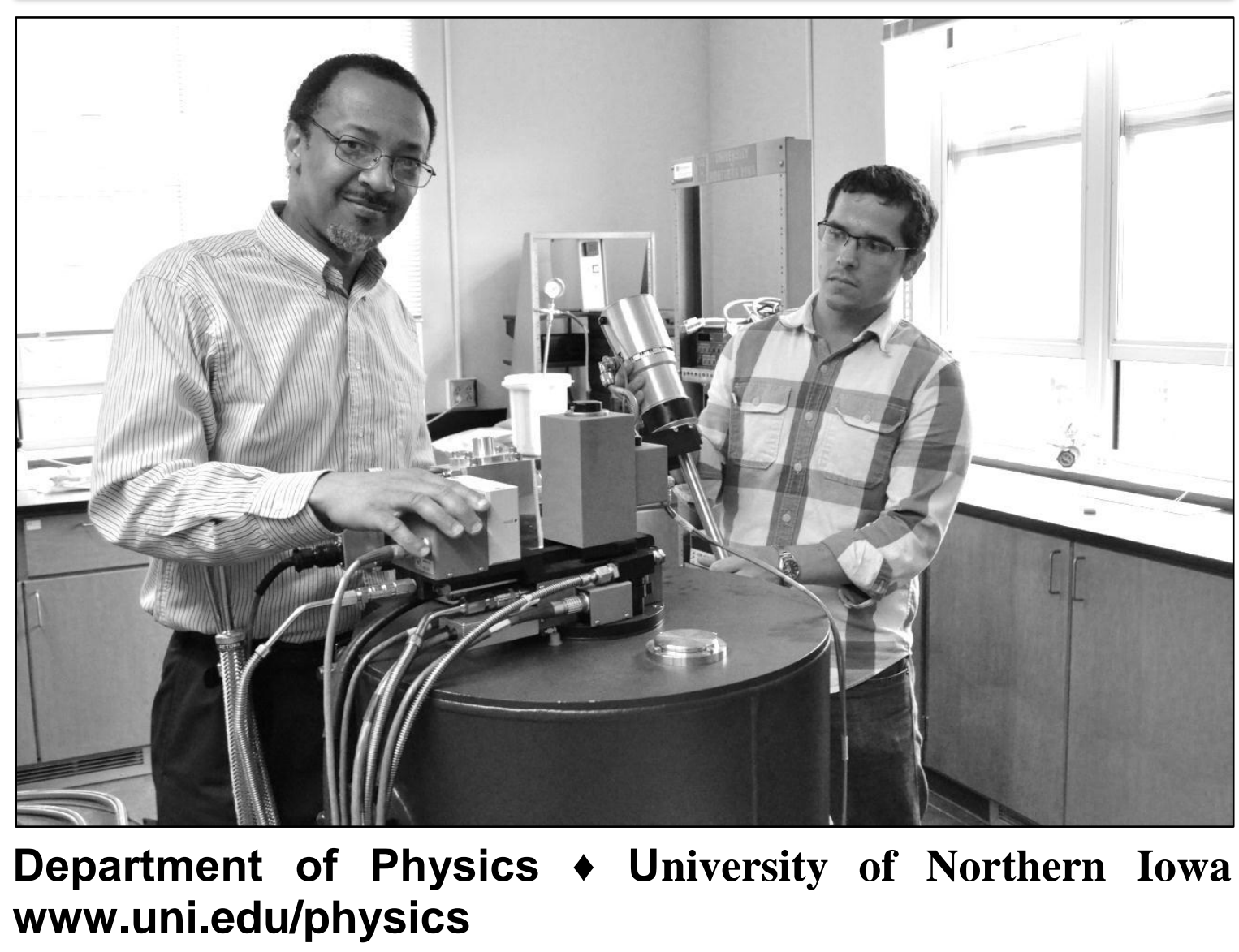

